\title{
Formation de la citoyenneté et communautés discursives
}

Jean-Paul Bernié

\section{(2) OpenEdition}

1 Journals

Édition électronique

URL : http://journals.openedition.org/trema/1705

DOI : 10.4000/trema.1705

ISSN : 2107-0997

Éditeur

Faculté d'Éducation de l'université de Montpellier

\section{Édition imprimée}

Date de publication : 1 octobre 1999

Pagination : 61-68

ISSN : 1167-315X

\section{Référence électronique}

Jean-Paul Bernié, « Formation de la citoyenneté et communautés discursives », Tréma [En ligne],

15-16 | 1999, mis en ligne le 01 octobre 1999, consulté le 19 avril 2019. URL : http://

journals.openedition.org/trema/1705; DOI : 10.4000/trema.1705

Ce document a été généré automatiquement le 19 avril 2019.

Trema 


\title{
Formation de la citoyenneté et communautés discursives
}

\author{
Jean-Paul Bernié
}

1 Assurer le rôle de la discipline « français » dans la construction du lien social exige une reformulation et une réorganisation de la problématique des " compétences », qui ne doit pas être abandonnée, mais qui, telle quelle, technicise le débat et fausse la vision des enjeux et du contexte socio-historique, lequel exige un double déplacement de la discipline ${ }^{1}$.

\section{Conception de la citoyenneté : une étape transitoire.}

2 Les débats et pratiques éducatives actuels sur la citoyenneté ont souvent du mal à se dégager d'un flou ou d'un réductionnisme où l'on peut voir un effet de la nature même de l'étape actuelle en la matière, transition entre une conception à dominante d'appartenance vers une conception à dominante instrumentale.

3 La citoyenneté traditionnelle reste affaire d'appartenance nationale. D'où son caractère abstrait, "étatique », renvoyant aux valeurs de la morale civique républicaine, mais enfermée dans sa dimension statutaire, délégataire, génératrice de passivité, désarmée face aux phénomènes d'exclusion sociale, à la crise du civisme et de l'idéologie de l'EtatNation, à la montée des particularismes.

4 A ces contradictions répond l'émergence d'autres modes d'implication dans l'espace public, effectifs et identitaires : citoyennetés locale et de proximité, multiculturelle voire européenne, et surtout active, faite de droits et d'obligations non hérités mais à construire dans des contextes diversifiés, par des voies excluant toute négation a priori des différences: l'enjeu identitaire de l'appartenance ne peut être négligé, mais reconstruit. N'est-elle pas ce qui permet à l'individu de se sentir «autorisé », capable, habilité ? On s'inscrit alors du côté du construit et non du convenu, du fondateur et non du fondé, mais dans un rapport dialectique. 
5 Le besoin se fait donc sentir d'une conception des apprentissages langagiers susceptibles de s'inscrire dans ce renouvellement et de l'outiller.

\section{Communautés discursives, concursus et translation}

6 La relation entre apprentissage de la langue et formation à la citoyenneté peut se déduire de la perspective suivante: "Les productions textuelles sont le medium au travers duquel se construisent les représentations collectives à partir desquelles sont situées et jugées les contributions individuelles à l'activité" (Bronckart, 1994, p. 385). Les savoirs à enseigner doivent permettre de décrire et d'instrumenter des pratiques langagières inscrites dans une perspective de concursus, non de consensus (expression de Francis Jacques). L'objectif est en effet d'aider l'élève à construire, à travers l'action langagière, son identité de sujet social d'une communauté à dimensions multiples; à intérioriser l'idée que son développement est conditionné par le fait de devenir "sujet entre les autres». Ces formules ne prétendent que résumer l'idée suivante : on ne peut se construire en tant que citoyen dans une communauté que si l'on peut se construire comme sujet dans plusieurs, sous peine d'enfermement dans la fausse citoyenneté d'une contre-communauté sociodiscursive comme celle du « rebeu » (verlan de «beur ») dont L.-J. Calvet a analysé le fonctionnement linguistique normatif et exclusif, négation du concursus, comme le serait, à l'opposé, le refuge dans le consensus a priori de la citoyenneté d'appartenance. Les aspirations multi-communautaires liées à l'idée de citoyenneté instrumentale s'opposent au particularisme; elles impliquent une mobilité à envisager en termes de translation plus que d'ascension, à l'intérieur d'un champ de valeurs à reconstruire.

7 Il semble donc pertinent de poser comme pierre angulaire de la démarche, sans se leurrer sur ce que peut l'école, mais en considérant la spécificité de son rôle :

- que la construction souhaitée passe essentiellement par un apprentissage « translatif » de la production langagière, permettant au sujet-élève de se construire dans un espace de tensions entre micro- et macro-communautés ;

- que le ciment de cette construction est l'intériorisation d'une conception triadique de la relation communicative, d'une posture d'énonciateur fondée sur une position de «transgrédience » (Bakhtine), d'exotopie, de relative extériorité.

La contribution de la discipline français à l'implication active de l'élève dans l'espace public passe donc par son institution comme sujet - producteur - dans une pluralité de communautés discursives. Nous entendons par «institution" un processus qui s'ancre d'abord dans ce que Bakhtine a appelé «compréhension responsive active» : ce qui, dans un processus communicatif réellement dialogique, fait de l'allocutaire un « coproducteur».

\subsection{Qu'est-ce qu'une « communauté discursive »?}

Il ne s'agit pas d'un découpage strict de la réalité sociale. La notion sert à caractériser le discours en tant que pratique sociale. Elle inclut :

- Des pratiques sociales présupposant et engendrant des normes d'échange : tout discours s'inscrit dans une institution (" pouvoir normatif assujettissant mutuellement les individus à certaines pratiques, sous peine de sanctions $»^{2}$ ), où il a valeur de mécanisme d'auto-contrôle de l'institution, fonction qui engendre des genres spécifiques (le compte rendu...). 
- Des valeurs cognitives imprégnant (et construites par) les productions langagières ayant pour cadre ces pratiques : « des critères d'acceptabilité rationnelle permettant de transformer en fait une donnée empirique, sous-tendus par des croyances en matière d'épanouissement cognitif humain. [...] Sans les valeurs cognitives, nous n'avons ni monde ni faits » (Putnam, 1984). Des critères de pertinence épistémique structurent ces valeurs, et génèrent du dicible en régulant toutes sortes d'opérations linguistiques.

- Des valeurs pragmatiques, concernant l'interaction sociale, et par conséquent la dimension rhétorique des productions langagières (Ber-nié, 1995). Une communauté discursive est aussi un espace de polémicité ${ }^{3}$ mis en scène par des genres précis, définissant le système des positions énonciatives possibles parce qu'assimilables à des rôles socialement identifiables entre lesquels le discours peut construire ses objets, espace où le sujet parlant pourra devenir « inter-personnel » et non impersonnel ou « personnel » au sens idéaliste d'antan. Sur cet arrière-plan on peut compléter la thèse de Bronckart: "C'est par (et dans) l'interaction verbale que se fabriquent les systèmes de représentations sociales (ou mondes représentés) qui définissent le contexte des actions humaines. Et c'est sur la base de cette connaissance langagière des mondes que sont évaluées les actions ascrites à un agent. [...] C'est là le premier sens $d u$ concept de médiation». Ces représentations «nationalisent» les communautés discursives en distribuant les rôles discursifs sur trois instances (locuteur, destinataire, et Tiers-Parlant). Ce schéma triadique ${ }^{4}$ est une donnée essentielle du problème. Sans référence au Tiers, la relation interlocutive "Je - Tu» échoue dans l'impasse irénique-agonistique. La construction d'une communauté discursive ne peut contribuer à celle de la citoyenneté que si elle s'opère au niveau du sujet dans un mouvement de mise à distance. Le Tiers appelle et fonde la recontextualisation: d'où l'intérêt de la notion de « transgrédience ».

\subsection{Les deux déplacements de la discipline « français ».}

11 L'école est une communauté discursive double: elle produit une culture d'apprentissage (les savoirs de la «mise à distance »...), mais elle ne peut le faire efficacement qu'au prix d'un métabolisme continuel avec les communautés, sources des pratiques auxquelles elle se réfère - sous peine d'opacifier les situations d'apprentissage. Mais son " ouverture » sur des pratiques sociales (qu'elle transforme...) doit être contrôlée en vertu de ses finalités propres. Une pratique d'apprentissage communicatif en projet n'a de sens pour l'implication du sujet dans l'espace public que si la définition de ce projet, sa mise en œuvre et sa gestion remplissent deux conditions.

\subsubsection{Reproduire la réalité sociale des échanges}

12 La première est qu'elle doit simuler les échanges pertinents dans un domaine socialement attesté, correspondant à un pan de "savoirs forts", de connaissances culturellement nécessaires, et permettent à l'élève de s'approprier, au cours de la réalisation de ce projet, les connaissances relatives à l'histoire, au fonctionnement social et communicatif du domaine. Même la production de textes poétiques peut s'intégrer à cette perspective si les lectures, visites, rencontres, etc., menées au cours du projet, permettent à l'élève de se confronter aux «fonctionnements réglés de l'institution littéraire » (J.-F. Halté), qu'il s'agisse des techniques d'écriture ou du fonctionnement social. La programmation ne peut plus dépendre d'objectifs d'outillage linguistique formel. L'élève-énonciateur doit 
pouvoir s'instituer sujet idéologique de la communauté où la situation l'invite à se situer pour pouvoir transformer les dispositifs langagiers en outils psychologiques.

La notion de «savoirs langagiers » doit donc être repensée dans le cadre de l'entrée dans des communautés discursives plurielles. Elle n'aura de sens que dans une approche sociohistorique, liant, dans l'activité, les trois aspects de la maîtrise des genres (au sens bakhtinien) :

- l'image de destinataire et son retraitement, mettant en jeu tous les paramètres de l'interaction sociale, notamment la finalité. Ici interviennent des compétences pragmatiques : mais il n'y aurait aucun sens à les couper de ce qui fait la réalité du « fond aperceptif de l'Autre ", à savoir, les contenus qui gagnent leur substance à travers les genres reconnus dans la sphère d'échange considérée, tel que le dicible des communautés discursives mobilisées par l'activité. Un élève du CM n'apprend pas à expliquer en imitant la structure formelle d'un prétendu « type » explicatif, mais, d'abord, en adhérant à un rôle, celui d'expliqueur, et en identifiant à partir de là la teneur des questions faisant énigme dans la communauté où il doit tenir ce rôle, puis les contenus à convoquer pour dissiper l'énigme, enfin les genres par lesquels le tout s'actualise ;

- les formes discursives multiples qui peuvent articuler les deux volets précédents et constituent donc la médiation sémiotique de l'activité langagière. Tel est le premier déplacement exigé de la discipline « français ».

\subsubsection{Instituer un sujet de la réalité scolaire}

14 La deuxième est qu'elle doit amener l'élève à s'instituer sujet d'une communauté «tierce » : la communauté scolaire. Il s'agit du deuxième déplacement de la discipline français, interdépendant du précédent, dont il tire son sens : aspect vital.

L'institution du sujet est incompatible avec toute réduction instrumentale du langage et de son apprentissage. Des expériences comme celle de Wertsch (1986) montrent que, lorsque l'élève reprend à son compte le langage d'une communauté discursive, il ne reprend jamais le squelette formel, le langage - instrument, mais d'abord les rôles discursifs. L'appropriation d'outils suppose que l'élève s'adresse à la fois les questions et les réponses, qu'il ait intériorisé plusieurs rôles, reconstruit " pour soi » les deux versants de l'interaction. Ce qui est transféré de l'inter- dans l'intra-psychologique, ce sont des formes sociales de comportement, c'est le début d'une culture. Le "Je" de l'élève s'instituant sujet n'est plus le «moi» de l'individu saisi dans sa dimension psychoaffective, mais le sujet "transgrédient" d'un espace social multi-communautaire. Nouvelle relative extériorité, suite de décontextualisations-recontextualisations successives. Ainsi peut et doit être défini l'un des paramètres souvent ignorés de toute production langagière : l'image d'énonciateur, réponse à la question prioritaire « En tant que QUI est-ce que je parle/écris », trop longtemps orientée à partir d'un point de vue solipsiste.

16 La question de l'enjeu « interne » au sujet révèle donc toute son importance. La rupture inévitable des situations d'apprentissage par rapport aux pratiques sociales réelles est un facteur d'opacité qui explique le mépris des contrats et le refus d'implication de nombreux élèves. A-t-on toujours bien pensé le traitement de ce décalage, impliquant le rapport au langage " ficelé » par les contextes sociaux d'origine, cantonnant le sujet dans la gestion immédiate de ses relations communicatives quotidiennes et inhibant ses capacités à prendre de la distance ${ }^{5}$ ? Transformer les représentations de ce que le sujet 
peut et croit pouvoir faire du langage, passe par la construction d'outils psychologiques lui permettant d'agir sur un nombre élargi de situations et de réguler son activité à l'aide de schèmes d'utilisation. Les déplacements discursifs sont à la fois effet et cause des capacités d'action. La question des savoirs langagiers est celle d'un outillage à inscrire dans ce cadre. Tel est le deuxième axe de déplacement requis de la discipline « français ».

\section{Quels « savoirs langagiers »?}

L'ordre scriptural (et non l'écrit au sens étroite ${ }^{6}$ ) peut être un outil privilégié du travail de la citoyenneté parce qu'il est le lieu privilégié de pratiques de recontextualisation dans une communauté élargie et multidimensionnelle: il permet de façonner des postures permettant au sujet d'habiter plusieurs communautés discursives. D'un point de vue ethnologique, dans les sociétés sans écriture ${ }^{7}$, les règles de la vie sociale sont régies et apprises par une logique d'action, sans pratique d'explicitation. Les connaissances existent comme immanentes aux personnes, groupes, situations et pratiques sociales qui en sont à l'origine. Alors que dans les aspects propres aux cultures d'écrit, apparaissent divers facteurs de germination de la «transgrédience », passant du « moi » au « je » : une détermination "contractuelle» mieux définie des rapports propres aux communautés discursives (un journal et son lectorat... ) ; un dépôt social mieux contrôlable des contenus convoqués et des opérations effectuables; la possibilité et le besoin de connaissances dépersonnalisées, et avec la constitution d'une communauté de destinataires éloignée, celle d'une position d'énonciateur distinct du simple particulier.

Les opérations intellectuelles que l'écrit rend possibles sont toujours en relation avec l'apparition de postures sociales. Ainsi, le langage écrit n'est pas "décontextualisé »: cette décontextualisation est en fait une "recontextualisation", l'entrée par rupture dans un nouveau contexte socio-langagier. Le passage de l'élève par une diversité de projets d'écriture finalisés comme entrée dans une communauté discursive jouera son rôle si les savoirs langagiers sont présentés en relation aux pratiques sociales de la communauté discursive simulée : on ne traitera plus la production d'écrits scientifiques à coups de modèles "typologiques» formels, mais en s'inspirant d'initiatives comme le " congrès de jeunes chercheurs » (Académie d'Amiens, 1998), ou les expériences de classe encouragées par D. Bucheton et J.-C. Chabanne (1998), où c'est par le biais d'un spectacle sur la Révolution que l'élève du Cours Moyen entre en tant qu'acteur dans Je dicible du champ historique, et se construit ainsi des outils langagiers et une posture d'historien ${ }^{8}$, l'un à travers l'autre. Et vice-versa: les recherches bordelaises montrent quels bonds qualitatifs sont possibles lorsque les «conflits socio-cognitifs » s'inscrivent dans des simulations de controverse scientifique, c'est-à-dire prennent en compte l'usage des documents, les systèmes de raisonnement et d'échange, les modes de constitution du fait scientifique, etc.

Cet aspect du déplacement de la discipline suppose donc que les situations d'apprentissage ne soient plus régies par des objectifs linguistiques au sens technique, mais par des objectifs culturels au sens complet: il est capital d'apprendre la « raison graphique » de quelques grands genres publics lorsque cela est appelé dans des situations permettant de construire un sujet « transgrédient » ou « interpersonnel ». 


\section{Conclusion générale}

La nature des savoirs nécessaires découle de la mise en relation de deux aspects : sur le plan historique, la connexité du développement d'une certaine culture d'écrit et d'une forme de lien social ; et, au niveau des individus et de leurs apprentissages, le pouvoir de l'ordre scriptural de fonctionner comme lieu privilégié de la construction outillée d'un rapport au langage indispensable pour l'institution du sujet.

21 Notre perspective impose de consommer la rupture avec les démarches dérivées de «l'objectivisme abstrait» critiqué par Bakhtine: ce qui nous est resté de l'héritage saussurien, avec sa configuration épistémologique qui aboutit, pour la phrase d'abord comme ensuite pour le texte, à administrer d'abord des «structures» alors qu'elles n'auraient au mieux de sens que comme outils de passage a posteriori entre ces « jeux de langage » chers à Wittgenstein, ces usages socialement situés qui sont source du sens. L'objet de l'enseignement du français, ce sont les "structures variationnelles de la langue » (A. Berrendonner, 1981), redéfinies aujourd'hui comme ce qui permet la reprisedéplacement (d'un genre, par un sujet). De même qu'il n'y a pas de "jeu de langage » transcendantal, il n'existe pas de savoirs langagiers in abstracto: la perspective plurinormaliste reposant sur une interaction dialectique entre composantes de la culture des communautés en présence, des fonctions et fonctionnements discursifs, et du « déjà là » de l'enfant, doit être repensée dans le cadre du processus de décontextualisation recontextualisation, donnant tout son sens à la mise à distance, à l'analyse et à l'intégration des codes sociaux: par conséquent, à des activités métalinguistiques repensées. Comprendre origine et fonctionnement des outils est une condition du mouvement de décontextualisation-recontextualisation, et l'on pourra reprendre ici les pistes proposées par Danielle Bouix-Leeman (" La grammaire ou la galère ») : instaurer la mise en controverse des règles de grammaire est une manière de construire à hauteur $\mathrm{du}$ sujet-élève l'indispensable épistémologie historique englobant les savoirs-outils (le code commun aux variations).

Il est fondamental pour la refondation de la citoyenneté que l'école ose enfin aborder de front la dimension cognitive et culturelle de l'accès à une position de sujet dans une pluralité de contextes socio-discursifs.

\section{BIBLIOGRAPHIE}

BAKHTINE M. : Esthétique de la Création Verbale. Paris, Gallimard, Collection Nrf, 1984.

BAUTIER E. et BUCHETON D. : « Interactions : co-construction du sujet et des savoirs ", in Le

Français Aujourd'hui « Interactions : dialoguer, communiquer ", № 113(Mars 1996).

BERNARDIN J. : Comment les enfants entrent dans la culture écrite. Paris, Éd. De Retz, 1997.

BERNIÉ J.-E : « Jeu des contextes et jeu des Je », in La communication. Toulouse, Presses

Universitaires du Mirail, 1996.

Tréma, 15-16 | 1999 
BERNIÉ J.-P : «Éléments théoriques pour une didactique interactionniste de la langue maternelle ", in BROSSARD M. et FIJALKOW J. (éds.) : Apprendre à l'école : perspectives Piagétiennes et Vygotskiennes. Bordeaux, Presses Universitaires de Bordeaux, 1998.

BROSSARD M. : « Un cadre théorique pour aborder l'étude des élèves en situation scolaire », in Enfance, Tome 46, № 4. 1992, pp. 189-200.

BROSSARD M., LABROILLE M., LAMBELIN G. et SABBAH D. : « Rôle du contexte dans les écrits scolaires ", in BARRÉ C. et De MINIAC (éds.) : Vers une didactique de l'écriture - Pour une approche pluridisciplinaire. Paris-Bruxelles, De Bœck Université, 1996.

BUCHETON D. et CHABANNE J.-C. : « Le point de vue, le doute et le savoir », in Le Français Aujourd'hui, № 123 (Septembre 1998), « Argumenter, enjeux et pratiques ".

DABÈNE M. : « L'adulte face à l'écrit », in Études de Linguistique Appliqué, № 59 (Octobre 1985).

JACQUES F. : « Consensus et conflit : une réévaluation », in Parrett H. (éd.) : La communauté en paroles. Communication, consensus, ruptures. Liège, Mardaga, 1991.

NONNON E. : « La notion de point de vue dans le discours », in Pratiques, № 100 (Décembre 1998).

PUTNAM H. : Raison, Vérité et Histoire. Paris, Ed. de Minuit, 1984.

WERTSCH J. : "The language experience of five year old children at home and at school", in cooKGOM-PERZ J. (éd.) : The social construction of Literacy. Cambridge, Cambridge University Press, 1986.

\section{NOTES}

1. Cet article est une version abrégée d'une analyse destinée à paraître dans l'ouvrage collectif faisant suite au colloque « Langage et Citoyenneté ».

2. BERRENDONNER A., 1981, p. 95 - J.P. Bronckart a proposé un classement en institutions économiques et sociales, étatico-politique, littéraire, académico-scientifique, de soin, de répression, scolaire, familiale, médiatique, de loisirs et de contact quotidien. Le statut des productions langagières varie de l'une à l'autre : telle peut être définie sur critères sociologiques, telle autre par les pratiques discursives qui s'y tiennent (les deux dernières).

3. Reprise-transformation d'un terme de Charaudeau - Cf., "Les espaces de parole dans la situation d'interlocution ", in Cahiers du Centre Interdisciplinaire des sciences du langage, № 10. Toulouse, Université de Toulouse-le-Mirail, 1994, p. 335 - espace axiologique inter-discursif où le sujet se construit par tension avec le «fond aperceptif » de son interlocuteur, en jouant sur le dialogisme intrinsèque du signe, sur sa « pluriaccentuation sociale » (Bakhtine).

4. Cf., sur ces notions - BERNIÉ : 1998, p. 171-180; et 1996.

5. $C f$., à ce sujet - BAUTIER et BUCHE TON : 1996.

6. Pour éviter la réduction d'un mode de production au seul « canal », se reporter aux travaux de Michel Dabène.

7. Ces réflexions sont redevables à la réinterprétation des travaux de Goody et Olson par Michel Brossard.

8. J.-F. Halté objecterait que cette posture n'échappe pas aux ambiguïtés qui gangrènent la rédaction scolaire: l'enfant y est-il élève-romancier ou romancier-élève? Tout dépend des conditions mises à la réalisation de la simulation scolaire : la classe est un espace du «faire comme si » et l'école une communauté discursive. La construction sémiotique de l'objet est un problème indissociable de celui de la position de sujet. 


\section{RÉSUMÉS}

Sur quel terrain déplacer aujourd'hui la discipline "français » pour tenter d'assurer son rôle dans la construction du lien social ? Celui de l'acculturation à l'écrit, vu comme lieu privilégié d'opérations visant à instituer et impliquer le plus grand nombre comme sujets de communautés discursives multiples et élargies. Les processus de décontextualisation - recontextualisation exigés conduisent à reformuler aussi bien les contenus de la discipline que les démarches didactiques.

Upon which field of knowledge should the "French language" school discipline be shifted nowadays in order to secure its rote in the construction of social bond building? Should it be that of acculturation to the written word, seen as the favored locus of mental operations having as their goal the organization and involvement of the largest number of people as subjects of a multiplicity of broadened communities of discourse? The requisite decontextualisation recontextualisation processes lead to the reformulation both of the contents of the discipline as well as of its didactic approaches.

\section{INDEX}

Mots-clés : acculturation, communauté discursive, contexte, institution du sujet

Keywords : community of discourse, context, organisation of the subject

\section{AUTEUR}

\section{JEAN-PAUL BERNIÉ}

IUFM d'Aquitaine, Université de Bordeaux II 